\title{
THE MOTION OF POINT VORTEX DIPOLE ON THE ELLIPSOID OF REVOLUTION
}

\author{
Sun-Chul Kim
}

\begin{abstract}
A pair of point vortices of the same strength but opposite sign is called a vortex dipole. We consider the limiting case where two vortices approach infinitely close while the ratio of the strength to the distance kept constant. The motion of such point vortex dipole on the ellipsoid of revolution is investigated geometrically to conclude that the trajectory draws a geodesic up to the leading order of perturbation, whose direction is determined by the initial orientation of the dipole. Related issues are also remarked.
\end{abstract}

\section{Introduction}

Dynamics of vortex is a crucial part of classical and modern fluid mechanics. The most basic vortex motion is probably that of point vortices in twodimension where the vorticity is ideally concentrated at a point. The interacting mechanisms of such point vortices are fundamentally important and helpful to understand the relevant physics of fluids. In spite of extensive study of dynamics of point vortices for centuries, there still remain many open issues [10].

In fact, we point out that even the two-vortex system, i.e., a pair of vortices display certain interesting motions. On the constant curvature surfaces (the plane, the sphere and the hyperbolic plane), Kimura [9] studied the motion of a pair of vortices with opposite strengths and argued that trajectories are always geodesic curves. Specifically, by stereographically projecting the sphere (and the hyperbolic plane) onto the two-dimensional plane, the dynamics of vortices is expounded indirectly. This method has an inevitable limitation since stereographic projection is only for the sphere (and the hyperbolic plane).

We here present an extension of this result by studying the point dipole motion on an ellipsoid of revolution (or symmetric ellipsoid) which is obtained by rotating an ellipse about its (shorter) axis. Recently the basic dynamical equations of point vortex on such ellipsoid of revolution are pursued in [3]

Received September 29, 2008; Revised November 11, 2008.

2000 Mathematics Subject Classification. Primary 76B47, 76M45, 53A17.

Key words and phrases. point vortex, ellipsoid of revolution, perturbation expansion, geodesic, vortex dipole.

This work was supported by the Chung-Ang University Research Grants in 2007. 
by a perturbation technique which will be used in this paper. In addition, instead of the stereographic projection, we adopt a direct geometrical approach and incorporate the parallel translation property of geodesic. More precisely, regarding the ellipsoid as imbedded in $\mathbb{R}^{3}$, we derive and analyze the dynamical equations of vortex directly and then check the parallel translation of the vortex dipole. The difficulty in generalizing the geodesic property of dipole paths for more general curved surfaces or even for general two-dimensional manifolds is then commented. A related conjecture in [8] turns out to be incorrect.

The current study has some meaning in the geophysical phenomena of global atmosphere and ocean circulation process. In fact, the physics of vortex dipole has been a central theme in various geophysical topics such as atmospheric jets [11], $\beta$-effect [4], vortex modon [7]. Also, in the viewpoint of dynamical systems, the vortex dipole is an important example of the ergodicity of vortex motion on certain smooth embedded ergodic surfaces [2] although most of point vortex systems are not ergodic [12].

\section{Vortex dipole on an ellipsoid of revolution}

First we specify the ellipsoid of revolution $E^{2}$ defined by

$$
E^{2}: \frac{x^{2}}{R^{2}}+\frac{y^{2}}{R^{2}}+\frac{z^{2}}{R^{2}(1+\epsilon)}=1,
$$

where $R>0$ and $\epsilon$ is a small number. The shape of $E^{2}$ is varying with $\epsilon$. More precisely, according to $\epsilon>0$ or $\epsilon<0$, the ellipsoid is prolate or oblate, respectively. And the corresponding eccentricity $e$ of the generating ellipse is given by $e=\sqrt{\epsilon /(1+\epsilon)}$ and $e=\sqrt{-\epsilon}$. This scaling produces the relation $\epsilon=O\left(e^{2}\right)$ between two parameters $\epsilon, e$ and therefore, the truncated first order system is expected to be a good approximation even for rather large values of the eccentricity.

On the ellipsoid $E^{2}$, let us consider $N$ point vortices located at

$$
\mathbf{x}_{1}=\left(x_{1}, y_{1}, z_{1}\right), \ldots, \mathbf{x}_{N}=\left(x_{N}, y_{N}, z_{N}\right)
$$

with vortex strengths $\Gamma_{1}, \ldots, \Gamma_{N}$, respectively. The corresponding Hamiltonian of the motion of the $N$-vortices up to the leading order in $\epsilon$ is expressed by

$$
H\left(\mathbf{x}_{1}, \ldots, \mathbf{x}_{N}\right)=\frac{1}{2} \sum_{i, j \neq k} \Gamma_{i} \Gamma_{j} \log \left(\mathbf{x}_{i}-\mathbf{x}_{j}\right)+\frac{\epsilon}{2} \sum_{i, j \neq k} \Gamma_{i} \Gamma_{j}\left(z_{i}^{2}+z_{j}^{2}\right),
$$

where $z_{i}, z_{j}$ are the $z$-components of $\mathbf{x}_{i}, \mathbf{x}_{j}$, respectively. (See [3] for derivation.) From this we derive the dynamical equations of point vortex on $E^{2}$,

$$
\dot{\mathbf{x}}_{i}=\sum_{j \neq i} \Gamma_{j} \frac{\mathbf{x}_{j} \times \mathbf{x}_{i}}{l_{i j}^{2}}+\sum_{j \neq i} \epsilon \Gamma_{j} \mathbf{x}_{i} \times z_{j} \mathbf{z},
$$

where $l_{i j}=\left|\mathbf{x}_{i}-\mathbf{x}_{j}\right|$ is the three dimensional Euclidean distance and $\mathbf{z}=(0,0,1)$ is the unit $z$-direction vector. 


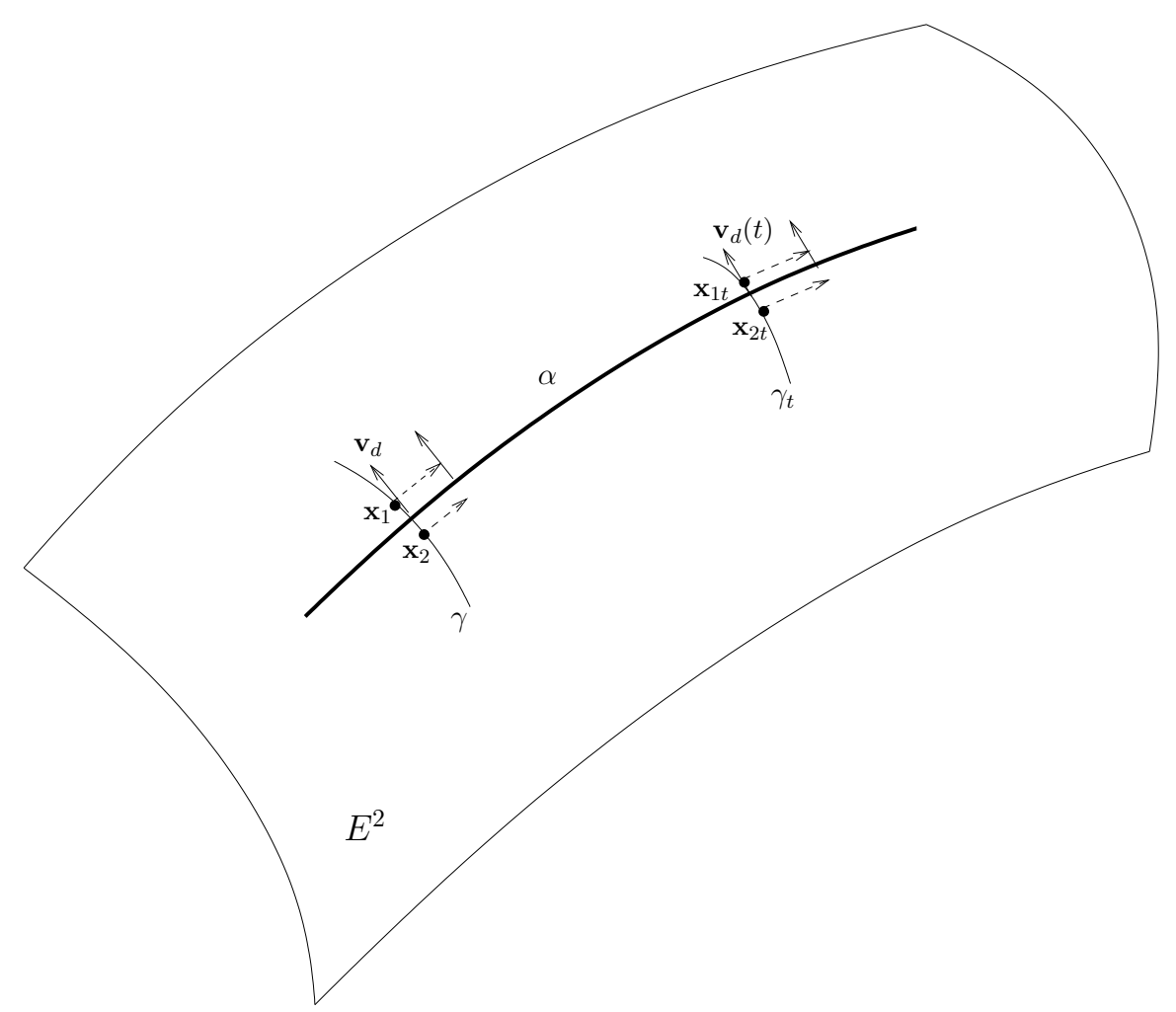

Figure 1. The motion of point vortex dipole transports $\mathbf{v}_{d}$ parallel in time

We then construct a point vortex dipole on $E^{2}$ as follows. Choose a point $\mathbf{x}_{d}$ on $E^{2}$ and a unit tangent vector $\mathbf{v}_{d} \neq \mathbf{0}$ in $\mathbb{R}^{3}$ at $\mathbf{x}_{d}$. We generate a point vortex dipole at $\mathbf{x}_{d}$ with a direction $\mathbf{v}_{d}$ as follows. (Here direction roughly means how two point vortices get close whose precise definition is given below.) First, we project $L=\left\{\tau \mathbf{v}_{d}:-1 \leq \tau \leq 1\right\}$ onto $E^{2}$ to obtain a curve $\gamma=\gamma(\tau)$ with $\gamma(0)=\mathbf{x}_{d}$ on $E^{2}$ and put the projected images of $\mathbf{v}_{d},-\mathbf{v}_{d}$ be $\mathbf{x}_{1}, \mathbf{x}_{2}$ on $E^{2}$. Then two point vortices $\mathbf{x}_{1}, \mathbf{x}_{2}$ approach to $\mathbf{x}_{d}$ along $\gamma$ forming a point vortex dipole at $\mathbf{x}_{d}$. Here, the convergence is performed along a fixed direction $\mathbf{v}_{d}$ which is the tangent of $\gamma$ at $\mathbf{x}_{d}$. From this we define the direction of a point vortex dipole by the unit tangent vector $\mathbf{v}_{d}$, which is related to the orientation of point vortex dipole (Figure 1). In this paper, we abuse the term vortex dipole for point vortex dipole for convenience because we deal the point dipole case only. 
Following this procedure, we choose two point vortices at $\mathbf{x}_{1}, \mathbf{x}_{2}$ with nonzero strengths $\Gamma,-\Gamma$, respectively. Then (1) reduces to

$$
\dot{\mathbf{x}}_{1}=\Gamma \frac{\mathbf{x}_{1} \times \mathbf{x}_{2}}{l_{12}^{2}}-\epsilon \Gamma \mathbf{x}_{1} \times z_{2} \mathbf{z}, \quad \dot{\mathbf{x}}_{2}=\Gamma \frac{\mathbf{x}_{1} \times \mathbf{x}_{2}}{l_{12}^{2}}+\epsilon \Gamma \mathbf{x}_{2} \times z_{1} \mathbf{z} .
$$

As in [9], we make a dipole by keeping the aspect ratio $\tau$ of the strength to the distance

$$
\frac{\Gamma}{d_{E^{2}}\left(\mathbf{x}_{1}, \mathbf{x}_{2} ; \gamma\right)}=\tau>0 \text { fixed }
$$

while $\mathbf{x}_{1}, \mathbf{x}_{2}$ both approaching to $\mathbf{x}_{d}$ in a manner described above. Here $d_{E^{2}}\left(\mathbf{x}_{1}, \mathbf{x}_{2} ; \gamma\right)$ represents the intrinsic distance between $\mathbf{x}_{1}, \mathbf{x}_{2}$ along the curve $\gamma$ on $E^{2}$. In other words, it is the arclength of $\gamma$ between $\mathbf{x}_{1}, \mathbf{x}_{2}$. (Note that $\mathbf{x}_{1}, \mathbf{x}_{2}$ moves to $\mathbf{x}_{d}$ along $\gamma$.) Let us check if $\mathbf{x}_{1}, \mathbf{x}_{2}$ transport $\mathbf{v}_{d}$ in parallel by inspecting the derivative of $\dot{\mathbf{x}}$ with respect to $d_{E^{2}}\left(\mathbf{x}_{1}, \mathbf{x}_{2} ; \gamma\right)$ at $\mathbf{x}_{d}$. More precisely we rewrite the expression

$$
\frac{\dot{\mathbf{x}}_{1}-\dot{\mathbf{x}}_{2}}{d_{E^{2}}\left(\mathbf{x}_{1}, \mathbf{x}_{2} ; \gamma\right)}=\frac{\epsilon \Gamma}{d_{E^{2}}\left(\mathbf{x}_{1}, \mathbf{x}_{2} ; \gamma\right)}\left[\mathbf{x}_{1} \times z_{2} \mathbf{z}-\mathbf{x}_{2} \times z_{1} \mathbf{z}\right]
$$

by adding and subtracting $\mathbf{x}_{1} \times z_{1} \mathbf{z}$ to the numerator into the form,

$$
\frac{\epsilon \Gamma}{d_{E^{2}}\left(\mathbf{x}_{1}, \mathbf{x}_{2} ; \gamma\right)}\left[\left(\left(\mathbf{x}_{2}-\mathbf{x}_{1}\right) \times z_{1} \mathbf{z}+\mathbf{x}_{1} \times\left(z_{2}-z_{1}\right) \mathbf{z}\right]\right.
$$

As we form the vortex dipole by letting $d_{E^{2}}\left(\mathbf{x}_{1}, \mathbf{x}_{2} ; \gamma\right) \rightarrow 0$, since $\frac{\Gamma}{d_{E^{2}}\left(\mathbf{x}_{1}, \mathbf{x}_{2} ; \gamma\right)}=$ $\tau>0$ fixed, we necessarily have

$$
\lim _{d_{E^{2}}\left(\mathbf{x}_{1}, \mathbf{x}_{2} ; \gamma\right) \rightarrow 0} \frac{\dot{\mathbf{x}}_{1}-\dot{\mathbf{x}}_{2}}{d_{E^{2}}\left(\mathbf{x}_{1}, \mathbf{x}_{2} ; \gamma\right)}=\mathbf{0}
$$

and established the parallel translation at the initial movement of vortex dipole.

Now, we consider the trajectory of the dipole $\alpha: \mathbf{x}_{d}(t), 0 \leq t \leq t_{0}$ on $E^{2}$ with $\mathbf{x}_{d}(0)=\mathbf{x}_{d}$ for some $t_{0}>0$ whose (local) existence is assumed. At each time $t$, we investigate the direction vector $\mathbf{v}_{d}(t)$ which is, by definition, transported by the dipole motion and defined along the trajectory with $\mathbf{v}_{d}(0)=\mathbf{v}_{d}$. Then the argument at $t=0$ above can be analogously repeated at $\mathbf{x}_{d}(t)$ with the direction $\mathbf{v}_{d}(t)$ and newly generated $\mathbf{x}_{1}, \mathbf{x}_{2}$ at $\mathbf{x}_{d}(t)$ (denoted by $\mathbf{x}_{1 t}, \mathbf{x}_{2 t}$ ) along $\gamma_{t}$ again transport $\mathbf{v}_{d}(t)$ parallel (Look at Figure 1 ). In summary,

Theorem 2.1. The direction vector $\mathbf{v}_{d}(t)$ of point vortex dipole moves parallel along the trajectory at any time. Then the point vortex dipole $\mathbf{x}_{d}(t)$ moves perpendicular to $\mathbf{v}_{d}(t)$ always.

Accordingly, from the first part of theorem above, the normal vector $\mathbf{v}_{d}(t)$ to $\alpha$ is parallel for all time. In other words, the tangent vectors to $\alpha$ are always parallel from the second part of the theorem, which is a geometric definition of a geodesic curve (See [5], p. 291). We remark that the sphere case $(\epsilon=0)$ is included during this argument to rediscover the result in [9]. The situation 
is dynamically equivalent to the roll of a small buggy with two same wheels joined by an infinitesimal axle (See [1] pp. 34-35).

Since our conclusion is only for the first order approximation of vortex dynamics, the next step of research will be inspecting the full exact trajectory of dipole by deriving the exact dynamical equations of point vortices on the ellipsoid of revolution. However, as commented in [3], the perturbation approach shows that the next order computations are too complicated and does not permit exact analytic solutions. Therefore, we might think of approximating solutions by certain numerical methods.

\section{Remarks on the general curved surface case}

Presumably, in regard to the present study, the final goal will be studying the trajectory of dipole on general curved surfaces or two-dimensional manifolds. The fundamental difficulty here is to set up the equations of motion of point vortices on general surfaces. This was initiated by Hally [6] who obtained a conformal coordinate formulation of vortex dynamics in the complex variables for the general simply connected surfaces. In [3], this approach is adopted and a perturbation series is calculated for the conformal factor function in the case of ellipsoid of revolution. As observed there, it is very hard to compute the conformal function in general except some highly symmetric surfaces such as a plane, a sphere, a circular cylinder, etc.

On the other hand, as we formulated in this paper, an alternative approach is possible. It is to regard the surface as imbedded in $\mathbb{R}^{3}$ and write the point vortex equations in three dimensional vector format. In particular, this approach produces the following formula for the velocity of $i$-th point vortex as in [8]

$$
\dot{\mathbf{x}}_{i}=\sum_{j \neq i} \Gamma_{j} \frac{\mathbf{n}_{j} \times\left(\mathbf{x}_{i}-\mathbf{x}_{j}\right)}{\left|\mathbf{x}_{i}-\mathbf{x}_{j}\right|^{2}}
$$

where $\mathbf{n}_{j}$ is the unit normal to the given surface at $\mathbf{x}_{j}$ for $i, j=1, \ldots, N$. (See Chapter 1 of [10] for details.) This representation successfully unifies the plane and the sphere vortex motions by taking $\mathbf{n}_{j}=(0,0,1)$ and $\mathbf{n}_{j}=\mathbf{x}_{j} / r$ respectively, where $r$ is the radius of the sphere. It is also implicitly conjectured on p. 147 in [8] if this formula is true for more general curved surfaces by taking the corresponding unit normal of the given surface $\mathbf{n}_{j}$ at $\mathbf{x}_{j}$. Of course, as this formula does not impose any condition for point vortices of staying on the specific surface, we should supplement an additional constraint that the vortex motion is performed on the given surface for all time.

However, by comparing with the formula (2), we realize that this is not the case for the ellipsoid of revolution. Let us illustrate the incorrectness of (3) by direct computation. For the dipole case, if (3) is correct, it should be

$$
\dot{\mathbf{x}}_{1}=\Gamma \frac{\mathbf{n}_{2} \times\left(\mathbf{x}_{1}-\mathbf{x}_{2}\right)}{l_{12}^{2}}, \quad \dot{\mathbf{x}}_{2}=\Gamma \frac{\mathbf{n}_{1} \times\left(\mathbf{x}_{1}-\mathbf{x}_{2}\right)}{l_{12}^{2}} .
$$


In the ellipsoid of revolution case, we put $R=1$ for simplicity and first expand

$$
\left\|\left(x_{2}, y_{2}, z_{2}(1+\epsilon)^{-1}\right)\right\|=\sqrt{1+z_{2}^{2}\left((1+\epsilon)^{-2}-(1+\epsilon)^{-1}\right)}=1-\epsilon \frac{z_{2}^{2}}{2}+O\left(\epsilon^{2}\right)
$$

since $\left(x_{2}, y_{2}, z_{2}\right)$ is on $E^{2}$. Thus the normal is given by

$$
\begin{aligned}
\mathbf{n}_{2} & =\frac{\left(x_{2}, y_{2}, z_{2}(1+\epsilon)^{-1}\right)}{\left\|\left(x_{2}, y_{2}, z_{2}(1+\epsilon)^{-1}\right)\right\|} \\
& =\left(\mathbf{x}_{2}-\epsilon z_{2} \mathbf{z}+\cdots\right)\left(1+\epsilon \frac{z_{2}^{2}}{2}+\cdots\right) \\
& =\mathbf{x}_{2}+\epsilon\left(-z_{2} \mathbf{z}+\frac{z_{2}^{2}}{2} \mathbf{x}_{2}\right)+O\left(\epsilon^{2}\right) .
\end{aligned}
$$

Substituting this into (4) produces

$$
\Gamma \frac{\mathbf{n}_{2} \times\left(\mathbf{x}_{1}-\mathbf{x}_{2}\right)}{l_{12}^{2}}=\Gamma \frac{\mathbf{x}_{2} \times \mathbf{x}_{1}}{l_{12}^{2}}+\epsilon \Gamma\left(\frac{z_{2} \mathbf{z} \times\left(\mathbf{x}_{1}-\mathbf{x}_{2}\right)}{l_{12}^{2}}+z_{2}^{2} \frac{\mathbf{x}_{2} \times \mathbf{x}_{1}}{l_{12}^{2}}\right)+O\left(\epsilon^{2}\right) .
$$

Since the two point vortices are staying on the ellipsoid during the whole motion, the instantaneous velocity should be confined in the tangent plane. In other words, we need to compute the projection of the velocity $\dot{\mathbf{x}}_{1}$ onto the tangent plane at $\mathbf{x}_{1}$. By the vector product with $\mathbf{n}_{1}$, we explicitly obtain the components of projected images. Expanding the results from (2) and (4) in the power of $\epsilon$, we derive the order of $\epsilon$ terms

$$
\epsilon \Gamma z_{2}\left(\mathbf{x}_{1} \times \mathbf{z}\right) \times \mathbf{x}_{1}
$$

and

$$
\epsilon \Gamma\left(\frac{z_{2} \mathbf{z} \times\left(\mathbf{x}_{1}-\mathbf{x}_{2}\right)}{l_{12}^{2}} \times \mathbf{x}_{1}+z_{2}^{2} \frac{\left(\mathbf{x}_{2} \times \mathbf{x}_{1}\right) \times \mathbf{x}_{1}}{l_{12}^{2}}\right)
$$

respectively, which obviously shows disagreement. In conclusion, although we are not sure if the formula (3) is true only for the planar and the spherical cases, we suppose that the formula is valid for very special surfaces of high symmetry.

\section{References}

[1] M. Berger, A Panoramic View of Riemannian Geometry, Springer-Verlag, Berlin, 2003.

[2] K. Burns and V. J. Donnay, Embedded surfaces with ergodic geodesic flows, Internat. J. Bifur. Chaos Appl. Sci. Engrg. 7 (1997), no. 7, 1509-1527.

[3] C. Castilho and H. Machado, The N-vortex problem on a symmetric ellipsoid: a perturbation approach, J. Math. Phys. 49 (2008), no. 2, 022703, 12 pp.

[4] M. T. Dibattista and L. M. Polvani, Barotropic vortex pairs on a rotating sphere, J. Fluid Mech. 358 (1998), 107-133.

[5] B. A. Dubrovin, A. T. Fomenko, and S. P. Novikov, Modern Geometry-methods and applications. Part I. Second edition. Graduate Texts in Mathematics, 93. SpringerVerlag, New York, 1992.

[6] E. Hally, Stability of streets of vortices on surfaces of revolution with a reflection symmetry, J. Math. Phys. 21 (1980), no. 1, 211-217. 
[7] D. Hobson, A point vortex dipole model of an isolated modon, Phys. Fluids A 3 (1991), no. $12,3027-3033$.

[8] R. Kidambi and P. K. Newton, Motion of three point vortices on a sphere, Phys. D 116 (1998), no. 1-2, 143-175.

[9] Y. Kimura, Vortex motion on surfaces with constant curvature, R. Soc. Lond. Proc. Ser. A Math. Phys. Eng. Sci. 455 (1999), no. 1981, 245-259.

[10] P. K. Newton, The N-Vortex Problem. Analytical Techniques, Applied Mathematical Sciences, 145. Springer-Verlag, New York, 2001.

[11] C. Snyder, D. Muraki, R. Plougonven, and F. Zhang, Inertia-Gravity waves generated within a dipole vortex, J. Atmos. Sci., to appear

[12] J. B. Weiss and J. C. McWilliams, Nonergodicity of point vortices, Phys. Fluids A 3 (1991), no. 5, part 1, 835-844.

Department of Mathematics

Chung-Ang University

SEOUL 156-756, KOREA

E-mail address: kimsc@cau.ac.kr 